\title{
Heat generation of core-shell particles composed of biodegradable polymer and iron oxide
}

\author{
C. Oka, K. Ushimaru, N. Horiishi*, T. Tsuge, and Y. Kitamoto \\ Interdisciplinary Graduate School of Science and Engineering, Tokyo Institute of Technology, 4259 Nagatsuta-cho, Midori-ku, \\ Yokohama 226-8503, Japan \\ *Bengala Techno Lab., 1-19 Kodai, Miyamae-ku, Kawasaki 216-0007, Japan
}

A suspension of core-shell particles composed of a biodegradable polymer core and iron oxide nanoparticles (IONPs) exhibits higher heat generation under an alternating magnetic field compared with a suspension of IONPs at the same IONP concentration. The improvement of the heat generation will be attributed to the change of the strength of dipolar interactions between adjacent IONPs. The IONPs in the core-shell particles were relatively isolated, in contrast with that in IONP suspension before the formation of the core-shell particles. Therefore, in the core-shell particles, the dipolar interaction is reduced, and the heat-generating capability is increased. This explanation is brought through investigating the influence of the agglomerate size of IONPs on the heat-generating capability.

Key words: magnetic hyperthermia, core-shell particle, iron oxide nanoparticle, dipolar interaction, hydrodynamic diameter, delta $M$ plots

\section{Introduction}

Magnetic hyperthermia (thermotherapy), which kills cancer cells by heat generated from magnetic nanoparticles under alternating magnetic fields, is a type of cancer treatment under study in clinical trials $^{1) \sim 4)}$. In the magnetic hyperthermia, tumor tissues are exclusively affected by selective accumulation of the magnetic nanoparticles, which are heat agents, in the tissues. Hence, the magnetic hyperthermia has been attracting significant attentions as a more effective treatment than conventional hyperthermia methods; a lot of researchers have been investigating magnetic nanoparticles and an apparatus generating the magnetic field for the treatment to improve the efficiency ${ }^{5)} 111$.

In our previous study, a core-shell particle composed of a core of a biodegradable polymer particle and a shell of assembly of magnetic iron oxide nanoparticles (IONPs) has been prepared as a candidate for a drug carrier for magnetically guided drug delivery system (MGDDS) ${ }^{12)}$. It can load hydrophobic drugs into its polymer core. We have already confirmed that the core-shell particles were strongly attracted to a $\mathrm{NdFeB}$ magnet, and will have magnetic properties enough for MGDDS.

In the present study, heat generation of the core-shell particles in the alternating magnetic field is investigated. The core-shell particle can act as a heat agent for magnetic hyperthermia as well as a MGDDS carrier because its structure includes magnetic nanoparticles. Therefore, the core-shell particle is expected to be a promising carrier having hybrid function of a drug carrier and a heat agent. The heat agent is an assembly of IONPs as a shell; the assembled structure of the IONP-shell is controlled by the

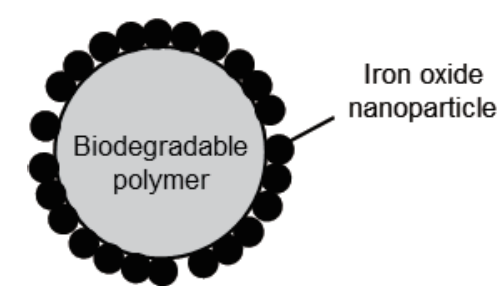

Fig. 1 Schematic illustration of core-shell particle composed of biodegradable polymer and iron oxide nanoparticles.

preparation conditions. The heat generation is induced by relaxation losses of IONPs, which are Néel and Brownian relaxation losses, and the relaxation dynamics is greatly affected by their agglomerate state due to dipolar interactions between IONPs ${ }^{13), 14)}$. To discuss the heat-generating capability of the core-shell particles in view of the agglomerate status in their suspension, the capability was compared with that of IONP suspensions with various agglomerate sizes manipulated by adding a flocculant and by changing IONP concentrations.

\section{Experimental}

Two types of IONPs, whose sizes were $8 \mathrm{~nm}$ and 11 $\mathrm{nm}$, were synthesized by a coprecipitation method using $\mathrm{FeCl}_{2}, \mathrm{FeCl}_{3}$ and $\mathrm{NaOH}$. Blocking temperatures of the IONPs were about $240 \mathrm{~K}$ for the $8 \mathrm{~nm}$ IONPs, and higher than room temperature for the $11 \mathrm{~nm}$ IONPs at a powder state. Although the temperature of the $11 \mathrm{~nm}$ IONPs indicates being ferromagnetic, both of the IONPs seem to be superparamagnetic in these suspensions at the room temperature. The core-shell particles were prepared as reported in our previous report with the 8 $\mathrm{nm}$ IONPS $^{12)}$. To evaluate the heat-generating 
capability, suspensions of the core-shell particles, the 8 $\mathrm{nm}$ IONPs, and the $11 \mathrm{~nm}$ IONPs were prepared at IONP concentrations of $0.1,0.3,0.5,1,1.5$, and $2.0 \mathrm{mg}$ $\mathrm{Fe} / \mathrm{mL}$. The IONP concentrations were determined by a concentration of $\mathrm{Fe}$ in the suspension. The agglomerate status, in particular, the size of the $11 \mathrm{~nm}$ IONPs was manipulated by adding polyvinyl alcohol (PVA) as a flocculant to the IONP suspension.

The heating curves of the suspensions were measured by a system with a coil generating the magnetic field at a frequency of $2 \mathrm{MHz}$ and a field strength of $3.4 \mathrm{kA} / \mathrm{m}_{\mathrm{rms}}$, and temperature was measured with a fiber-optic thermometer. Since the frequency is considerably high, it is presumed that the Neel relaxation is dominant rather than the Brownian relaxation in the condition. The heat-generating capability was evaluated by the Box-Lucas equation ${ }^{15)}$ :

$$
T(t)=T(0)+A\left(1-e^{-B t}\right)
$$

where $T$ is temperature, $t$ is time, and $A$ and $B$ are parameters related to the saturation temperature and the curvature of the heating curve. At $t=0$, the product $A \times B$ represents an initial rate of temperature rise $\Delta T$ $\Delta t$. The specific absorption rate (SAR) is proportional to the product, expressed as

$$
S A R=(\Delta T / \Delta t)\left(C / m_{\mathrm{Fe}}\right) \propto A B
$$

Table 1 Hydrodynamic diameter and the product $A \times B$ of fitting parameters for suspensions of $8 \mathrm{~nm}$ IONPs, and core-shell particles prepared using the $8 \mathrm{~nm}$ IONPs.

\begin{tabular}{ccc}
\hline & $\begin{array}{c}\text { Hydrodynamic } \\
\text { diameter } \\
(\mathrm{nm})\end{array}$ & $\begin{array}{c}A \times B \\
\text { per Fe mass } \\
(\mathrm{K} /(\mathrm{s} \cdot \mathrm{g} \mathrm{Fe}))\end{array}$ \\
\hline 8 nm IONPs & 79 & 37 \\
Core-shell particles & - & 64 \\
\hline
\end{tabular}

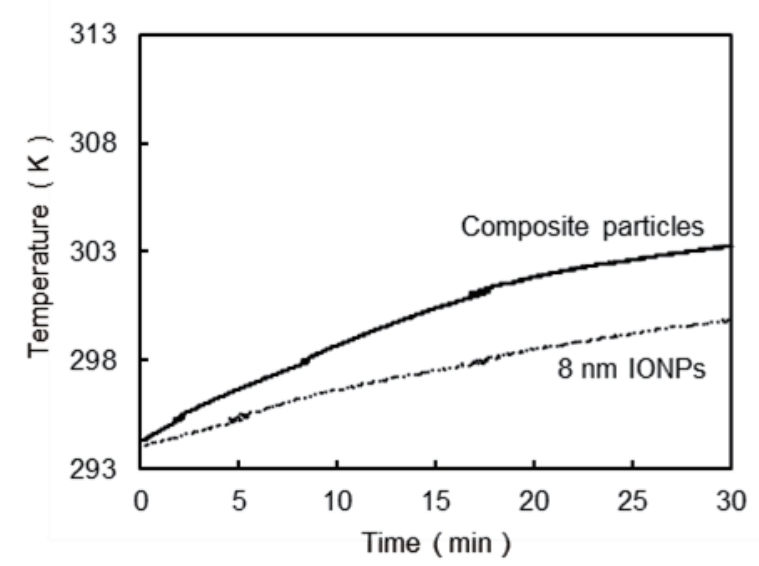

Fig. 2 Heating curves of $0.3 \mathrm{mg} \quad \mathrm{Fe} / \mathrm{mL}$ of suspensions of $8 \mathrm{~nm}$ IONPs, and core-shell particles prepared using the $8 \mathrm{~nm}$ IONPs. where $C$ is the heat capacity per unit mass of a specimen and $m_{\mathrm{Fe}}$ is the Fe mass per unit mass of a specimen. Therefore, the values of $A \times B$ were calculated from the heating curves of the suspensions of the core-shell particles and the IONPs as a substitute for SAR.

To elucidate the difference in magnetic interaction between IONPs and the core-shell particles, zero-field cooled (ZFC) and field cooled (FC) magnetization, isothermal remanent magnetization (IRM) and direct current demagnetization (DCD) of the $8 \mathrm{~nm}$ IONPs and the core-shell particles using the IONPs were measured. The ZFC and FC measurements were conducted at 8.0 $\mathrm{kA} / \mathrm{m}$ from $5 \mathrm{~K}$ to $320 \mathrm{~K}$. The IRM and DCD were measured at $5 \mathrm{~K}$ for delta $M$ plots $^{16)}$. The IRM was obtained by the following protocol; 1) starting from a state of zero remanence, 2) applying a field, and 3) measuring magnetization $\left(M_{\mathrm{r}}\right)$ after decreasing the field to zero. For the DCD, the protocol was 1) saturating the specimen in a positive field, 2) applying a negative field, and 3) measuring magnetization $\left(M_{\mathrm{d}}\right)$ after increasing the field to zero. $M_{\mathrm{rS}}$ and $M_{\mathrm{ds}}$ were obtained by changing the applying field in each process. The magnitude of the applying fields was from $12.0 \mathrm{kA} / \mathrm{m}$ to $638 \mathrm{kA} / \mathrm{m}$, and the field for the saturation was $798 \mathrm{kA} / \mathrm{m}$. The delta $M(\Delta M)$ plots can be obtained by the following equation $^{16)}$;

$$
\Delta M=M_{\mathrm{d}}-\left(1-2 M_{\mathrm{r}}\right)
$$

The $\Delta M$ expresses the degree of a deviation from a system without any magnetic interaction between magnetic nanoparticles. Generally, negative $\Delta M$ represents dipolar-like (demagnetizing) interactions, and positive $\Delta M$ indicates exchange-like (magnetizing) interactions.

\section{Results and discussion}

The capability of a suspension of the core-shell particles was superior to that of the $8 \mathrm{~nm}$ IONPs prepared at the same IONP concentration, which was $0.3 \mathrm{mg} \mathrm{Fe} / \mathrm{mL}$. Figure 2 shows heating curves of the suspensions, and Table 1 lists the product $A \times B$ calculated from the fitting parameters of these curves. The product was approximately doubled by the formation of the core-shell particles. We speculated this improvement would be induced by the change of the agglomerate state of IONPs. Indeed, the agglomerate state of IONPs in its suspension seemed considerably different from that in the core-shell particles. In the IONP suspension, IONPs agglomerate, resulting in the formation of a secondary particle; the hydrodynamic diameter measured by dynamic laser scattering (DLS) was $79 \mathrm{~nm}$. On the other hand, the IONPs on the core-shell particles relatively exist with a gap and the assembled size is equal to the size of the core-shell particle as apparently observed in a TEM image of Fig. 3. 
Therefore, we focus on agglomerate state, in particular, on the agglomerate size of IONPs as a parameter to strongly influence the heat generation in order to discuss the above-mentioned improvement of the capability in the core-shell particles. The $11 \mathrm{~nm}$ IONPs were employed for this experiment instead of the $8 \mathrm{~nm}$ IONPs to obtain larger amount of the heat generated from IONPs. The agglomerate size was manipulated by adding PVA. We selected a hydrodynamic diameter in the suspensions measured by DLS as a parameter strongly correlated with the agglomerate size. Open circles in Fig. 4 represent the hydrodynamic diameter of the $11 \mathrm{~nm}$ IONPs at PVA concentration of $0,0.1,0.2,0.5$, and $1.0 \mathrm{wt} \%$. The diameter was increased with the increase of PVA concentration. The IONP concentration was $0.3 \mathrm{mg}$ $\mathrm{Fe} / \mathrm{mL}$.

Figure 5(a) shows heating curves of the IONP suspensions with different concentrations of PVA. The curves were changed by an amount of PVA. However, it was found that the heat generation under the alternating magnetic field was observed even in PVA

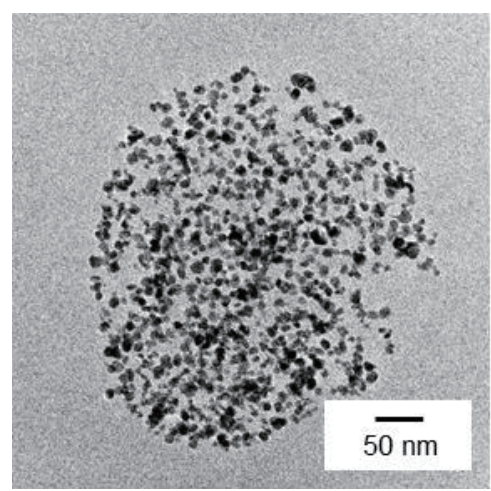

Fig. 3 TEM image of core-shell particle prepared using $8 \mathrm{~nm}$ IONPs.

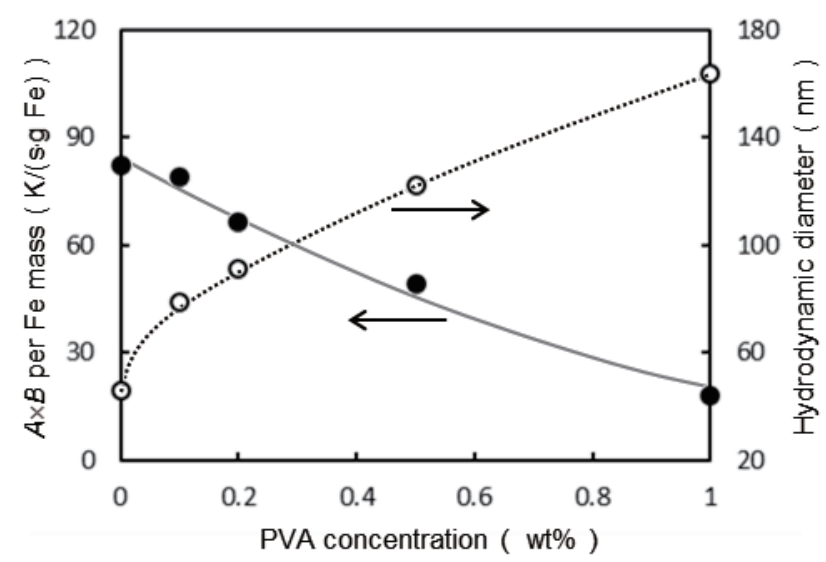

Fig. 4 Dependences of the product $A \times B$ (filled circles) and hydrodynamic diameter (open circles) of $11 \mathrm{~nm}$ IONPs on PVA concentration of the IONP suspension. aqueous solutions (aq.) without the IONPs as shown in Fig. 5(b). The heating of the PVA aq. would be caused by an eddy current loss ${ }^{17), 18)}$. The temperature rise depends on the PVA concentration. Therefore, we drew new curves subtracting the temperature rise of PVA aq. from heating curves of IONP suspensions, so as to estimate the heat generation induced only by IONPs. Figure 5(c) shows the recalculated curves, and filled circles in Fig. 4 indicate the products $A \times B$ of these fitting parameters. The product was decreased with the
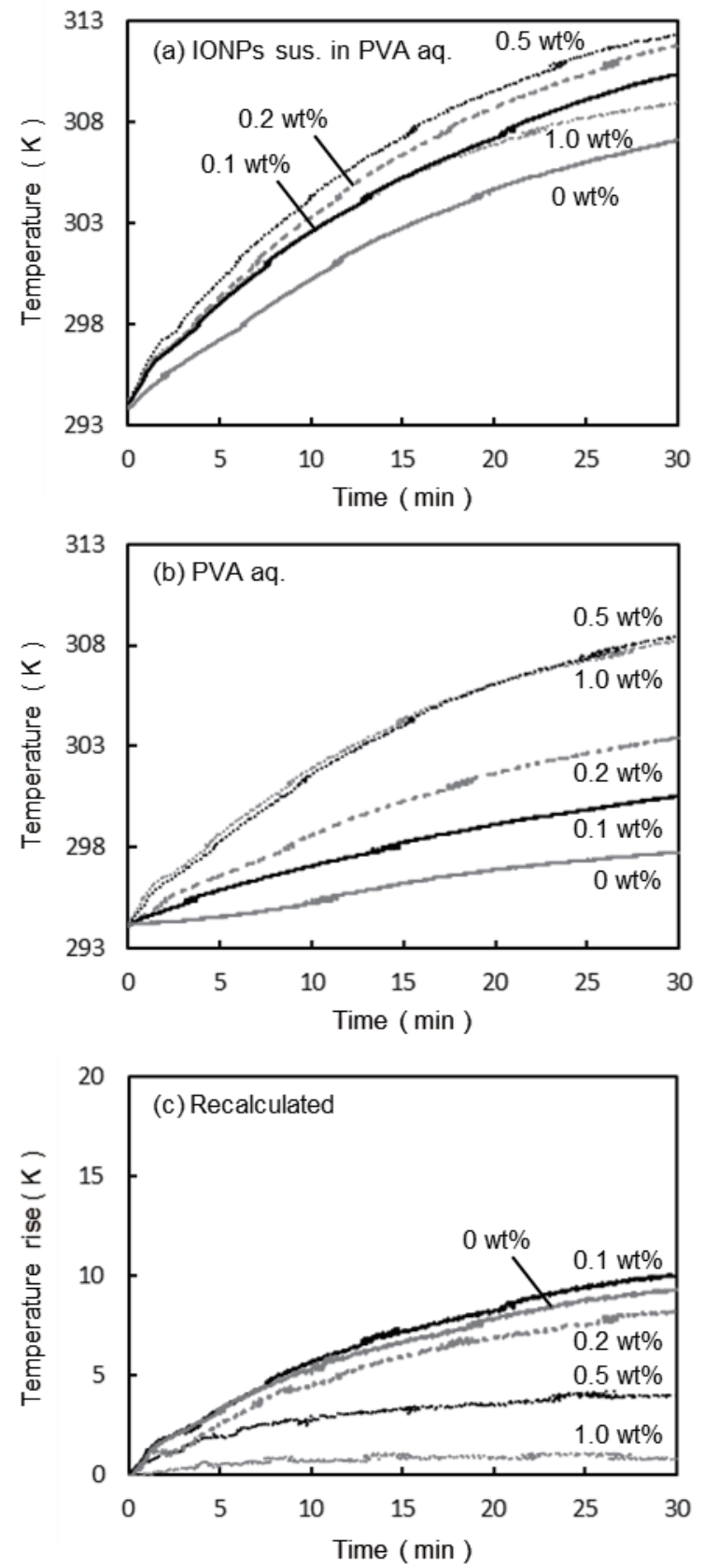

Fig. 5 Heating curves of (a) IONP suspensions prepared at different PVA concentrations, (b) PVA aqueous solutions at different concentrations without IONPs, and (c) curves recalculated by subtracting (b) from (a). 
increase of the PVA concentration. This result suggests that the heat-generating capability is reduced by the agglomeration.

To corroborate the results about the negative influence of the agglomeration of IONPs on the heating capability, the heating capability of IONP suspensions with different IONP concentrations were evaluated. The agglomerate size of IONPs can be regulated not only by adding the flocculant such as PVA but also by changing the concentration of IONPs in the suspension based on DLVO theory. Hence, the IONP suspensions were prepared at different IONP concentrations. The hydrodynamic diameter was increased with the increase of the concentration as shown in Fig. 6 (open circles). These heating curves after removing the heating effect of the solvent are shown in Fig. 7. The curves were drawn by subtracting the heating of water, which is the same as the result of 0 wt\% PVA aq. in Fig. 5(b). The product of the fitting parameters of the recalculated curves is indicated in Fig. 6 (filled circles). The product decreased exponentially with the IONP concentration. The product $A \times B$ is proportional to SAR. Therefore, this result indicates that the heat-generating capability of IONPs is reduced by the agglomeration induced by increasing the concentration of IONPs. The decrease of SAR at high concentrations has also been demonstrated in other papers ${ }^{13), 19), 20)}$.

Figure 8 represents the relationship between the hydrodynamic diameter of IONPs and the product of the fitting parameters; the relationship was obtained from the dependences of the hydrodynamic diameter and the product on the PVA concentration and IONP concentration seen in Figs. 4 and 6 . The product obviously exhibits a linear decrease against the increase of the diameter, indicating the reduction of the heating capability with the increase of the agglomerate size. The tendency has also been reported by other researchers although the reason has been unclear ${ }^{21)}$. In addition, the relationship from the PVA-concentration dependence (open circles) emerged above that from the IONP-concentration dependence (filled circles). Because the hydrodynamic diameter of IONPs with PVA was evaluated to be larger than that without PVA due to the existence of PVA, which was a hydrophilic polymer.

The reduction of the capability was probably related to dipolar interactions between adjacent IONPs in the agglomerate. Some researchers have reported that the dipolar interaction has a negative influence on SAR, and three dimensional cluster of magnetic nanoparticles exhibited the reduction of the heat generation ${ }^{22), 23)}$. We speculate the reason why the interaction will become greater with the increment of the agglomerate size of IONPs in the following. One IONP in a larger agglomerate interacts magnetically with more other IONPs than that in a smaller agglomerate, resulting in the increase of dipolar interactions. Therefore, the heat generation was reduced with the increase of the agglomerate size of IONPs leading to the increase of

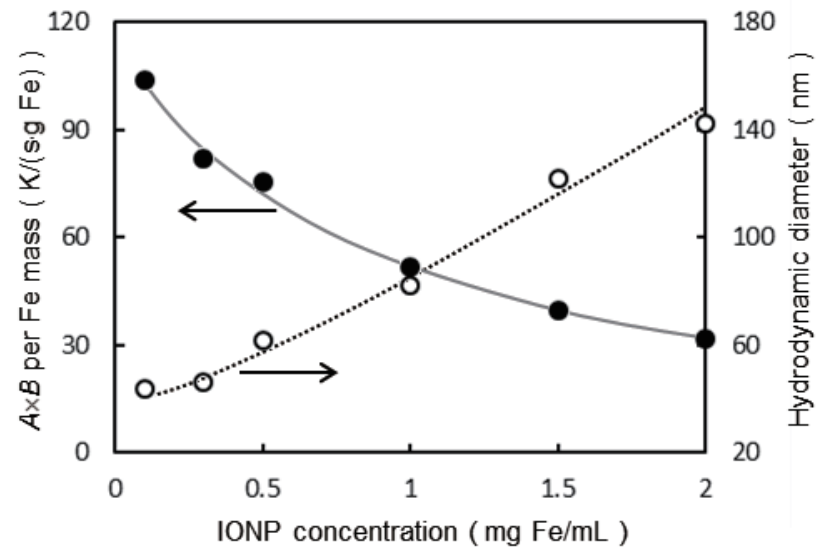

Fig. 6 Dependences of the product $A \times B$ (filled circles) and hydrodynamic diameter (open circles) of $11 \mathrm{~nm}$ IONPs on IONP concentration of its suspension.

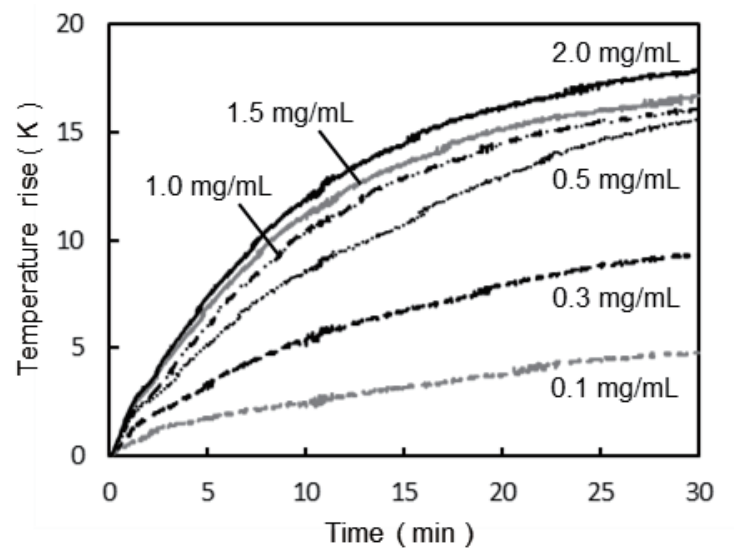

Fig. 7 Heating curves of IONP suspensions of different IONP concentrations after removing the heating effect of the solvent, that is the curves subtracting temperature rise of water.

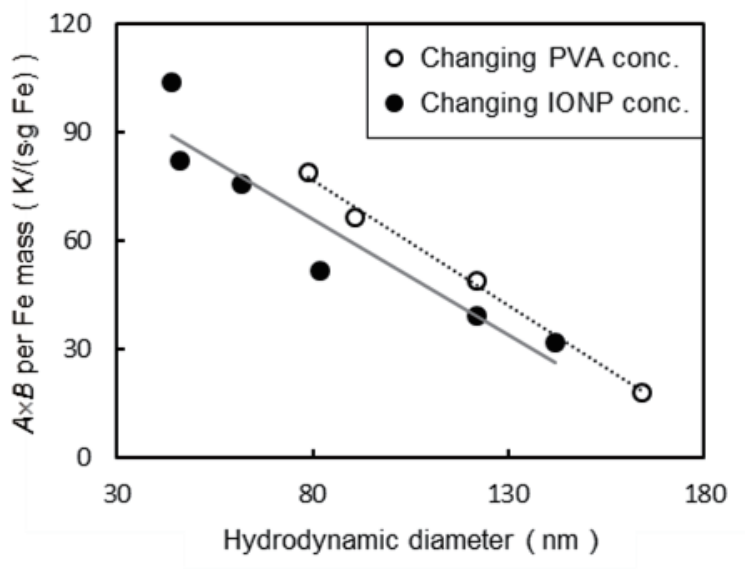

Fig. 8 The product $A \times B$ as a function of hydrodynamic diameter of IONPs in its suspensions changing PVA concentrations (open circles) and IONP concentrations (filled circles). 
number of IONPs which one IONP interacted with.

We speculate that the improvement of the heat-generating capability by the formation of the core-shell particles seen in Fig. 2 and Table 1 was induced by a change of their agglomerate state of IONPs. The IONPs were deposited on the biodegradable polymer core with a gap between each IONP in the core-shell particles. Whereas the assembly of IONPs is a two-dimensional cluster in the core-shell particles, the agglomerate of IONPs is a three-dimensional cluster in the IONP suspension, because the IONPs spontaneously agglomerate in order to reduce surface energy. The number of IONPs interacting with one IONP in the core-shell particles is less than that in the agglomerate of IONPs, which is the three-dimensional cluster of IONPs. Thus, dipolar interactions in the core-shell particles are weaker than in the agglomerate of IONPs, resulting in the improvement of the heat-generating capability. Figure 9 shows ZFC-FC curves of the core-shell particles and the $8 \mathrm{~nm}$ IONPs. It indicates that magnetic property of the core-shell particles is obviously different from the $8 \mathrm{~nm}$ IONPs.

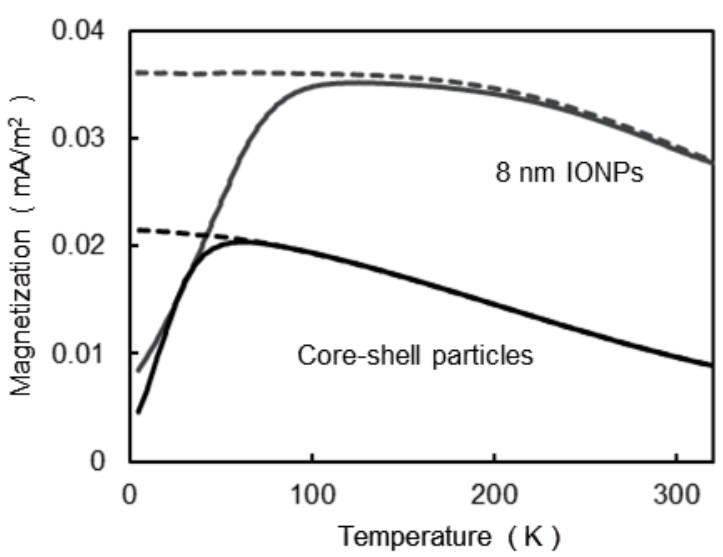

Fig. 9 ZFC (solid line)-FC (dotted line) curves of core-shell particles and $8 \mathrm{~nm}$ IONPs.

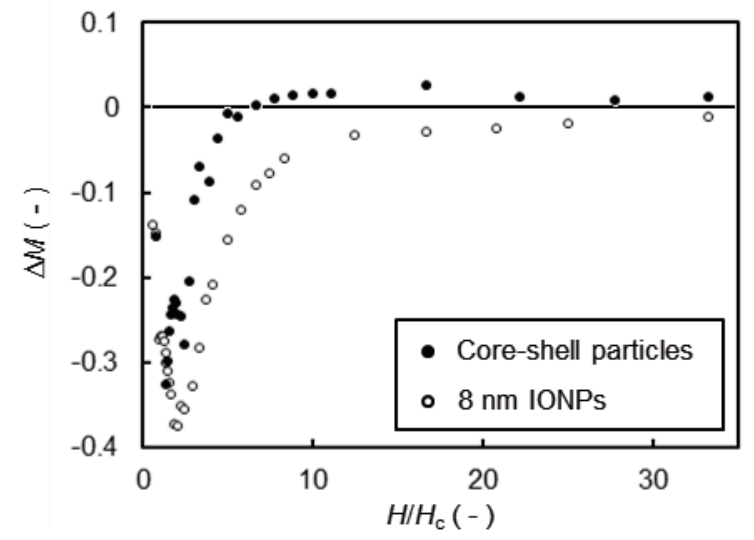

Fig. 10 Delta $M$ plots for core-shell particles and 8 $\mathrm{nm}$ IONPs. $H_{\mathrm{c}}$ is coercivity at $5 \mathrm{~K}$.
An energy barrier to magnetization reversal in the $8 \mathrm{~nm}$ IONPs seems to be larger in consequence of strong agglomeration. Figure 10 exhibits delta M plots obtained from IRM and DCD measurements ${ }^{16)}$. The magnitude of $\Delta M$ represents the degree of the magnetic interaction, and it is always zero in a system of non-interacting particles according to the Wohlfarth relationship. The maximum of the deviation from zero in $\Delta M$ was -0.33 for the core-shell particles, which was smaller than -0.38 for the $8 \mathrm{~nm}$ IONPs. The magnetic interaction, that is probably the dipolar interaction, was restrained by the formation of the shell assembly of the IONPs.

\section{Conclusion}

The heat-generating capability of the core-shell particles composed of the biodegradable polymer core and the iron oxide nanoparticles was discussed in the present paper. The capability of the core-shell particles was superior to that of the original IONPs used for the formation of the core-shell particles. The improvement by the formation of the core-shell particles were explained in view of the agglomerate state of magnetic IONPs correlating to dipolar interaction. We demonstrated that the heat-generating capability is inversely proportional to the hydrodynamic diameter, in other words, the agglomerate size of IONPs in the suspension. The dependence on the agglomerate size is probably explained by the number of IONPs interacting in the agglomerate. Finally, we concluded the higher capability of the core-shell particles was brought from the difference in the agglomerate state of IONPS between the core-shell particles and the original IONP suspension.

Acknowledgement The present work was partially supported by Kobayashi International Scholarship Foundation.

\section{References}

1) Q. A. Pankhurst, J. Connolly, S. K. Jones, and J. Dobson: J. Phys D, 36, R167 (2003).

2) R. Hergt, R. Hiergeist, I. Hilger, W. A. kaiser, Y. Lapatnikov, S. Margel, and U. Richter: J. Magn. Magn. Mat., 270, 345 (2004)

3) B. Thiesen, and A. Jordan: Int. J. Hyperthermia, 24, 467 (2008).

4) A. Jordan, R. Scholz, P. Wust, H. Fahling, and R. Felix: $J$. Magn. Magn. Mat., 201, 413 (1999).

5) C. P. Gooneratne, M. Kakikawa, T. Ueno, and S. Yamada: J. Magn. Soc. Jpn,. 34, 119 (2010).

6) S. Vasseur, E. Duguet, J. Portier, G. Goglio, S. Mornet, E. Hadova, K. knizek, M. Marysko, P. Veverka, and E. Pollert: J. Magn. Magn. Mat., 302, 315 (2006).

7) S. Maenosono, and S. Saita: IEEE Trans. Magn., 42, 1638 (2006).

8) B. Mehdaoui, A. Meffre, J. Carrey, S. Lachaize, L. M. Lacroix, M. Gougeon, B. Chaoudret, and M. Respaud: $A d v$. Funct. Mat., 21, 4573 (2011). 
9) S. Balivada, R. S. Rachakatla, H. Wang, T. N. Samarakoon, R. K. Dani, M. Pyle, F. O. Kroh, B. Walker, X. Leaym, O. B. Koper, M. Tamura, V. Chikan, S. H. Bossmann, and D. L. Troyer: BMC cancer, 10, 119 (2010).

10) L. M. Lacroix, R. B. Malaki, J. Carrey, S. Lachaize, M. Reapaud, G. F. Goya, and B. Chaudret: J. Appl. Phys., 105, 023911 (2009).

11) C. C. Berry, and A. S. G. Curtis: J. Phys. D, 36, 198 (2003).

12) C. Oka, K. Ushimaru, N. Horiishi, T. Tsuge, and Y. Kitamoto: J. Magn. Magn. Mat., 381, 278 (2015).

13) E. Lima, E. D. Biasi, M. V. Mansilla, M. E. Saleta, M. Granada, H. E. Troiani, F. B. Effenberger, L. M. Rossi, H. R. Rechenberg, and R. D. Zysler: J. Phys. D, 46, 045002 (2013).

14) A. Roch, Y. Gossuin, R. N. Muller, and P. Gillis: J. Magn. Magn. Mat., 293, 532 (2005).

15) M. Kallumadil, M. Tada, T. Nakagawa, M. Abe, $P$. Southern, and Q. A. Pankhurst: J. Magn. Magn. Mat., 321, 1509 (2009).

16) C. B. Andujar, D. Ortega, P. Southern, Q. A. Pankhurst, and N. T. K. Thanh, Nanoscale, 7, 1768 (2015).
17) S. A. Theron, E. Zussman, and A. L. Yarin, Polymer, 45, 2017 (2004).

18) F. Liu, S. Crozier, H. Zhao, and B. Lawrence, Concept. Magnetic Res., 15, 26 (2002).

19) C. M. Boubeta, K. Simeonidis, A. Makridis, M. Angelakeris, O. Iglesias, P. Guardia, A. Cabot, L. Yedra, S. Estrade, F. Peiro, Z. Saghi, P. A. Midgley, I. C. Leboran, D. Serantes, and D. Baldomir: Sci. Rep., 3, 1652 (2013).

20) A. Urtizberea, E. natividad, A. Arizaga, M. Castro, and A. Mediano: J. Phys. Chem., 114, 4916 (2010).

21) X. Wang, H. Gu, and Z. Yang: J. Magn. Magn. Mat., 293, 334 (2005).

22) L. C. Branquinho, M. S. Carriao, A. S. Costa, N. Zufelato, M. H. Sousa, R. Miotto, R. Ivkov, and A. F. Bakuzis: Sci. Rep., 3, 2887 (2013).

23) D. Serantes, K. Simeonidis, M. Angelakeris, O. C. Fesenko, M. Marciello, M. P. Morales, D. Baldomir, and C. M. Boubeta: J. Phys Chem., 118, 5927 (2014).

Received Oct. 20, 2015; Revised Feb. 19, 2016; Accepted Mar. 30,2016 